inpatient unit, of whom three had died after a drug overdose within the first four months after discharge. We successfully interviewed 103 patients (at a mean interval of 8.7 months after discharge). A search of records indicated that the remaining 29 patients were still alive one year after discharge.

To test whether loss of tolerance increased the risk of overdose, we grouped the patients into three categories, according to their opiate tolerance at the point of leaving treatment: 43 "still tolerant" (ST) patients who failed to complete detoxification; 57 "reduced tolerance" (RT) patients who completed the prescribed phase of detoxification but who prematurely left the treatment programme; and 37 "lost tolerance" (LT) patients who completed the detoxification and also completed the inpatient treatment programme.

The three overdose deaths that occurred within four months after treatment were all from the LT group; the two deaths unrelated to overdose (although both these patients had relapsed) were one LT patient with end stage renal failure and one RT patient with Clostridium welchii infection; no deaths occurred in the ST group (Fisher's exact test, $\mathrm{df}=2, \mathrm{P}=0.02$ ). This clustering did not derive from differences in duration to the follow up interview (mean durations were 9.5 months (ST), 8.7 months (RT), and 7.8 months (LT)).

We also considered length of time in treatment as a continuous variable. The five patients who died had stayed longer in the inpatient unit (mean 24.6 days (SD 7.6)) than the other 132 patients (15.6 days (8.1)) $(t=2.44, \mathrm{P}=0.02)$ (table). We looked for distinctive premorbid characteristics among the patients, all men, who died-possible clinical markers of risk of mortality after detoxification. Before admission these patients were more likely than the other patients to have been living alone, to have been taking higher doses of methadone, and to have been using heroin less often. They stayed longer in the inpatient unit and were more likely to have completed the treatment programme.

\section{Comment}

Patients who "successfully" completed inpatient detoxification were more likely than other patients to have died within a year. No patients who failed to complete detoxification died. Heroin addicts are known to have excess mortality. ${ }^{4}$ However, on the basis of previously published data we would have expected that in our group only one or two patients would have died within a year and only one from overdose. ${ }^{5}$ The clustering of the deaths from overdose in the group of patients who had successfully completed treatment is counterintuitive and illogical-unless it derives from loss of tolerance and consequent unpredictability of resumed heroin use. This study urgently requires replication, and if its results are confirmed these will need to be addressed within existing inpatient, residential, and custodial and associated aftercare programmes.

We thank the patients and staff of Wickham Park House, Bethlem Royal Hospital, South London and Maudsley NHS Trust.

Contributors: IS conceived the analysis of data from the follow up study designed by DB, JB, MG, and JS. TB and SR collected and entered the data. Statistical analysis was by JMcC and JS. JS and $\mathrm{JMcC}$ wrote the original draft, and all authors contributed to interpretation and revision. JS and DB are the guarantors. Funding: Henry Smith Charitable Foundation.

Competing interests: None declared.

Ethical approval: South London and Maudsley ethical committee.

1 Advisory Council on the Misuse of Drugs. Reducing drug-related deaths. London: Stationery Office, 2000.

Capelhorn J. Deaths in the first two weeks of methadone treatment in NSW in 1994: identifying cases of iatrogenic methadone toxicity. Drug Alcohol Reu 1998:17:9-17.

3 Bird SM, Hutchinson SJ. Male drugs-related deaths in the fortnight after release from prison: Scotland, 1996-99. Addiction 2003;98:185-90.

4 Oppenheimer E, Tobutt C, Taylor C, Andrew T. Death and survival in a cohort of heroin addicts from London clinics: a 22-year follow-up study. Addiction 1994;89:1299-308.

5 Farrell M, Neeleman J, Griffiths P, Strang J. Suicide and overdose among opiate addicts. Addiction 1996;91:321-3.

(Accepted 30 January 2003)

\title{
Changing prescription patterns for lithium and valproic acid in old age: shifting practice without evidence
}

Kenneth I Shulman, Paula Rochon, Kathy Sykora, Geoffrey Anderson, Muhammad Mamdani, Susan Bronskill, Chau T T Tran

Department of Psychiatry,

Sunnybrook and

Women's College

Health Sciences

Centre, Faculty of

Medicine,

University of

Toronto, Toronto,

ON, Canada

M4N 3M5

Kenneth I Shulman

professor

continued over

BMJ 2003;326:960-1
Over the past decade, valproic acid (prescribed as divalproex in North America) has been marketed as an alternative to lithium for treating bipolar disorders. For elderly patients, however, there is no clear evidence that valproic acid is more beneficial than lithium. Moreover, the evidence for the superiority of valproic acid in treating bipolar disorders-mixed episodes and rapid cycling-has been challenged in a recent Cochrane review. ${ }^{1}$ Valproic acid has not benefited patients with manic and psychiatric symptoms in dementia, despite the growing use of the drug in the management of these conditions. $^{2}$ Recently, the relatively rapid shift in prescription patterns has been questioned. ${ }^{3}$ We describe trends in the use of lithium and valproic acid in a large population of people over 65 .

\section{Methods and results}

We obtained information on drug use from the Ontario Drug Benefit Program, which provides comprehensive drug benefits to all residents aged 65 or older in Ontario, Canada. We identified all patients who had been taking lithium or valproic acid between 1993 and 2001 (prevalent users) and we further identified those patients who had not previously taken lithium or valproic acid (new users). We restricted our study to patients aged 66 or more to enable us to examine their previous drug use for a minimum of one year. Using unique encrypted health card numbers, we linked data on this cohort to two other large datasets-the Canadian Institute for Health 


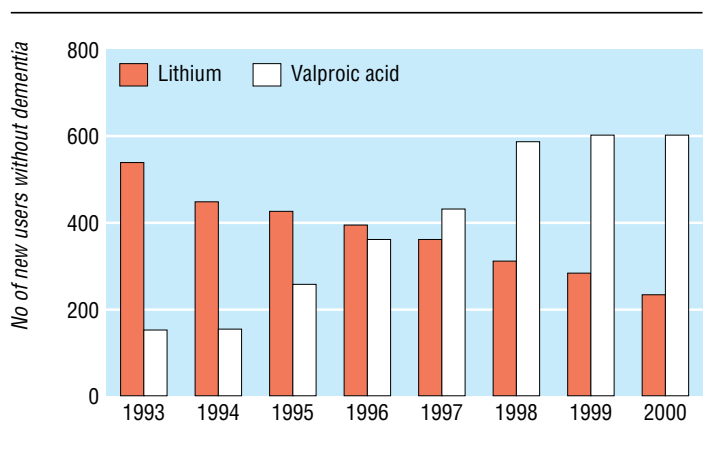

Year

New elderly users of valproic acid and lithium who did not have a diagnosis of dementia before the incident use, Ontario, 1993-2000

Information dataset of all hospital separations and the Ontario Health Insurance Plan dataset of all claims for physician services. These datasets provided information on hospitalisations and visits to physicians that helped us distinguish between psychiatric and anticonvulsant uses of lithium and valproic acid and between bipolar disorder and other indications such as dementia.

Among patients who had no previous history of convulsive disorders, we identified 3902 patients who had started taking lithium and 5341 patients who had started taking valproic acid between 1993 and 2001. New valproic acid users were slightly older than lithium users (75.4 years $v 73.5$ years). More than three quarters of new lithium users and fewer than two thirds of valproic acid users had contact with a psychiatrist. Almost one quarter of the new lithium patients and $41 \%$ of new valproic acid users had had a diagnosis of dementia.

The number of new lithium users per year fell from 653 older adults in 1993 to 281 in 2001, whereas the number of valproic acid users rose from 183 in 1993 to 1090 in 2001. The trend was similar when we eliminated patients who had had a diagnosis of dementia (figure). The number of new valproic acid users surpassed new lithium users in 1997, with a steady decline in new lithium users and a steady increase in new valproic acid users between 1993 and 2000.

\section{Comment}

Prescription patterns have shifted in favour of valproic acid over lithium, for elderly patients with bipolar disorder. This shift is occurring in the absence of evidence based data. Lithium carbonate has been a mainstay for the management of bipolar disorders for all age groups, but elderly patients pose a special concern because of the potential for toxicity. ${ }^{4}$ Before the use of lithium is abandoned for these patients, however, adequate evidence that valproic acid offers a comparable or superior efficacy, effectiveness, and safety profile is needed. Indeed, the concerns about lithium toxicity may reflect a failure to adjust for pharmacokinetic and pharmacodynamic factors that occur in old age. ${ }^{4}$ For example, renal clearance changes notably with age, and hence also lithium clearance. Better guidelines for dosage and serum concentrations of lithium carbonate for elderly people are needed before clinicians switch to prescribing new agents, especially with the associated risk of relapse that follows the discontinuation of lithium. ${ }^{5}$ Given the complexity, morbidity, and mortality associated with bipolar disorders and dementia in elderly patients, the use of mood stabilisers and other psychotropic agents requires ongoing systematic evaluation.

Contributors: KIS conceived the study, reviewed the literature, and wrote the initial draft. PR helped to conceive the study, revised the initial and subsequent drafts, and was overseer of the research network. KS analysed the data and developed the figure. GA, MM, $\mathrm{SB}$, and CT contributed to the study design, data analysis, and revisions of the manuscript. KIS is the guarantor.

Funding: This work was supported by the Canadian Institutes of Health Research Chronic Disease New Emerging Team programme (NET 54010).

Competing interests: None declared.

1 Macritchie KA, Geddes JR, Scott J, Haslam DR, Goodwin GM. Valproic acid, valproate and divalproex in the maintenance of bipolar disorder. Cochrane Database Syst Rev 2001;(3):CD003196.

2 Sival RC, Haffmans J, Jansen P, Duursma SA. Sodium valproate in the treatment of agoressive behaviour in patients with dementia randomized placebo controlled clinical trial. Int $J$ Ger Psychiatry randomized $p$

3 Dinan TG. Lithium in bipolar mood disorder. BMJ 2002;324:989-90.

4 Johnson G. Lithium-early development, toxicity, and renal function. Neuropsychopharmacology 1998;19:200-5.

5 Baldessarini RJ, Tondo L, Viguera AC. Discontinuing lithium maintenance treatment in bipolar disorders: risks and implications. Bipolar Dis 1999;1(Pt 1);17-24.

(Accepted 13 January 2003)
Institute for Clinical Evaluative Sciences (ICES), Sunnybrook and Women's College Health Sciences Centre

Kathy Sykora biostatistician Geoffrey Anderson senior adjunct scientist

Muhammad Mamdani

scientist

Susan Bronskill scientist

Chau T T Tran doctoral candidate

Kunin-Lunenfeld Applied Research Unit, Baycrest Centre for Geriatric Care, Toronto, $\mathrm{ON}$ Canada M6S 2E1

Paula Rochon assistant director Correspondence to: K I Shulman ken.shulman@sw.ca

\section{One hundred years ago}

\section{Degrees and how not to give them}

There was an Irishman once who explained that how he fell out of a third floor window did not matter; the important thing was what he reached at the bottom. The views of Sir William Ramsay in an essay on degrees in a recent number of the Contemporary Review are precisely the reverse; the degree a man reaches is nothing, how he gets there everything. According to him, outside university circles in England a man with a degree is regarded as one to be avoided if he present himself as a business recruit, whereas in Germany and America he is welcomed. This result he ascribes to the systems on which the degrees are awarded in the respective countries. The method of England he utterly condemns, because training for examinations results in men who have knowledge of the subjects on which they are examined, but no original ideas about them whatever. If the stories of some examiners are true, however, this is not always the case. Another statement is that the best men often fail to get through. This will meet with some assent, especially from those whose expressed views have been so original that they have failed to get examiners to accept them.

The fatal feature about English examinations appears to be the co-operation of outside examiners. They have an unpleasant way of asking questions to which candidates cannot supply answers, because they have not been taught them. This, of course, is dreadfully inconsiderate to the teachers, and in Germany and America such inconvenient persons are not invited to attend. (BMJ 1903;i:804) 